\title{
Transitioning a Marquee Orientation and Transition Program for Increased New Student Engagement and Retention Amidst the COVID-19 Pandemic
}

\author{
Devin Carpenter, University of Montana
}

One mid-sized public flagship institution in the Mountain West was forced to abandon a brand-new orientation and transition program, which had contributed to the first increase in first-time student retention in a decade. As educators worldwide were faced with similar challenges, three main concerns arose for this school: cancellation of in-person instruction and events indefinitely in March 2020, a long-term downward trend in enrollment and retention, and a lack of adequate space to host in-person activities. This paper discusses how these obstacles were overcome and shares the critical lessons learned as we look toward fall 2021.

Keywords: extended orientation, student success, COVID-19, retention

Higher education institutions exist in constant flux, continuously adapting to changing student needs, new research, fiscal demands, and myriad other compounding factors. While most orientation, transition, and retention (OTR) professionals are comfortable in this realm, none of us were prepared to confront the sudden, dramatic, and likely permanent changes the COVID-19 pandemic forced us to implement in spring 2020. Not only were students asked to finish their school year using, to this point, fairly uncommon remote technology, but we as OTR professionals had to adjust our plans to transition the next class of students to campus via remote technologies at arguably the most critical time in their journey.

Pre-matriculation and orientation plans are often years in the making and hinge on high-touch, in-person connections and engagement with the campus community to facilitate the highest possible yield (Secore, 2018). When enrollments across the country are trending down (St. Amour, 2020), our institution has lost more students in the previous decade than any other public flagship university (Bauman \& Brown, 2019). However, just when success was in sight, we faced a challenge unlike any we could have imagined.

In fall 2019, our institution, a mid-size public flagship in the Northwest, rolled out a completely re-imagined new student orientation program. This retention-focused extended orientation model was one of many factors that led to an almost 2\% increase in the first-to-second-year retention rate from fall 2019 to fall 2020 and the highest rate since 2016. This model was and still is, poised to become a marquee program in the region and establish our institution's onboarding process as one that emphasizes individual attention, community-wide engagement, and student success.

After a more in-depth review of the program's history and background, this paper discusses three main challenges and corresponding solutions implemented for the 2020 cohort of new students. In addition, we share strategies implemented to increase student engagement throughout the summer and fall and the many creative 
solutions we continue to use even now. Finally, lessons and recommendations for the future are discussed in hopes that readers can adapt them to fit student and institutional needs as the world continues to grapple with the lasting effects of the COVID-19 pandemic.

\section{Institutional and Programmatic History}

As noted previously, our university faced significant financial troubles directly related to a decade-long enrollment decline. A committee formed in late November 2018 was tasked with overhauling the fall 2019 orientation program to better meet the needs of a changing student body and to address ongoing challenges in the enrollment and retention of new students.

\section{Developing a Three-Part Transition and Orientation Program for Increased Student}

\section{Retention}

In concert with other retention and persistence measures, the committee developed a three-pronged approach: (a) early registration and business days in the spring, (b) a week-long extended orientation program consisting of an experiential community immersion component, and (c) a more traditional social and academic component. Together, these programs sought to help students build more connections in their new community, foster peer networks, and enhance their preparation for academic success. These outcomes are widely credited with significantly increased student success and retention. As a collective, these three programs form the mandatory new student orientation (NSO) implemented for all new first-year students entering in fall 2019. Initial assessment findings were encouraging, and the program was adopted as the new standard for OTR at our institution.

Before this systematic overhaul, students received no personal attention or engagement with the university until June, when new students and their families attended a two-day orientation program. During the two-day program, students took care of all the business of enrollment (e.g., register for courses and address outstanding financial aid concerns) while also trying to make peer connections, engage with their academic college, and learn about their new home and responsibilities. Students had no substantive interactions with the local community, and there was minimal welcome week activity when students arrived in August. The changes implemented in fall 2019 shifted the institutional and cultural mindset of what it means to be a student at the university. Not until recently has recruitment, retention, and enrollment been seen as a universitywide imperative and certainly not part of the overall retention and persistence efforts. The success of the new orientation model signaled a campuswide shift in thinking and demonstrated the possibilities when radical collaboration is expected and nurtured.

\section{Part 1: Spring On-Campus Registration Days}

The first piece of the program is early registration days, developed to allow students to take care of the essential items needed for enrollment: obtaining a student ID and first-semester course schedule. Having taken these steps, students can rest assured that they have their desired classes scheduled and truly focus on orienting themselves to their new home when they attend the other portions of the NSO program. In addition, as an institution without an enrollment deposit or formal admissions acceptance, this program allowed the campus to have early indicators of the size of our incoming class.

Five of these events were scheduled on Fridays beginning in April and continuing through the end of June. Due to overwhelming success, we added a sixth date to accommodate more students. As a campus with a high Pell-recipient population, we recognized that a physical trip to campus could pose a financial burden on many 
families, so an online version was developed to meet the needs of any student who could not or chose not to make the trip to campus. As planning began for the fall 2020 cohort, we doubled the registration day program dates to 10. Between opening registration in February 2020 and the closure of our campus in March, we had registered more than 300 students for these programs.

\section{Part 2: The Community Immersion Experience}

The next phase of our new orientation program is the community immersion experience, which encompasses the first half of the on-campus extended orientation week in August. The first day included the official move-in day for all students and the start of campus meal plans. This change is significant because, until this year, students had been required to attend the orientation and to pay extra for room and board. Overall, the vision of the immersion experience was to engage students with the institution and the local community while creating an opportunity for students to build connections with both current and incoming students. To do this, we created five categories from which students could choose-outdoors; entrepreneurship; science and technology; the arts; and diversity, service, and leadership-for immersion experiences in the local and campus community. Students were then placed into groups of 20 peers based on their preferences. A student ambassador (our version of an orientation leader) and an on-site representative from the host organization led the groups, which engaged in site-specific experiences for a total of nine hours across three days. Experiences include community service projects (e.g., trail work, invasive plant removal), team-building exercises, ecology work, entrepreneurship competitions, art installations, diversity and privilege workshops, biology experiments, and more.

In total, we partnered with more than 40 campus and community organizations. After the initial success, we were already receiving inquiries in early 2020 from new community partners who wanted to host the immersion experience. We even had a donor lined up to create a new overnight experience that would be free for a group of 20 students. The momentum of the program and buy-in from local community groups were tangible. We are still working with these partners to maintain interest while waiting for it to be safe enough to resume such largescale community-wide programming.

\section{Part 3: The Social and Academic Transition to College}

Finally, the second half of the on-campus orientation week focuses on social and academic expectations and the transition to campus. After completing their community immersion experience, students were sorted into new small groups based on meta-majors. We intentionally introduced students to peers with whom they shared a common interest (their community site) and those they would likely encounter in their classes to increase the possibility of connecting with others. The schedule for this portion of the week mimicked the traditional class day. The goal here was to create an early college experience and mitigate any surprises during the first few weeks of class. We retained two pieces of the former orientation program: bystander intervention training and the Student Production, a program that acts out fairly common social situations college students encounter and the various ways in which they can navigate them appropriately. These, along with peer-led, small-group discussions, addressed the social transition to college.

In addition to these topics, informational sessions were overhauled, and class tracks were established. Students attended two 50-minute lecture sections and one two-hour lab section, all based on their intended major. The purpose here was to bring students within academic areas together, get them in contact with faculty right away, and establish academic expectations. We developed 11 different class tracks, encompassing 58 individual lectures and labs provided by faculty across two days, to accommodate the fall 2019 cohort. 
Never in the history of our orientation program were faculty involved to this extent, let alone in high-touch ways like providing an early college mini-mester. In just one year, we redefined the narrative of what it means to become a student here and engaged the entire campus and surrounding community in the orientation and transition process for new students for the first time. We were finally positioning ourselves to increase the overall enrollment of our school for the first time since 2012.

\section{COVID-19 Challenges and Solutions Implemented}

As with many colleges around the country, ours faced the challenge of moving to entirely remote instruction in spring 2020. Concerns about retaining the incoming class were as significant as those for retaining continuing students. Plans were made to convert campus space to classrooms to facilitate as much in-person learning as possible when students returned for the fall 2020 semester, presenting another set of challenges for an orientation program designed to accommodate more than 1,000 students at once. Some of these obstacles were more easily navigated, while others required more significant innovation, campus collaboration, careful planning, and risk mitigation.

\section{Campuses Move to Entirely Remote Instruction and Close for the Semester.}

Like many colleges and universities, our institution operates on a semester system with fall and spring terms in addition to several summer session offerings. During the spring term, students have a week-long spring break that often falls in March. With the harsh reality of just how serious the COVID-19 pandemic was becoming last spring, our administration decided, like many schools did, to transition all in-person instruction online so that students would not need to return to campus after spring break. All campus events and programs were also required to move to a virtual format or be canceled. The big unknown at this point was how long this move would be necessary. At first, we decided to cancel the four April registration day sessions, hoping that by May we would be able to bring students and their families to campus. Eventually, we canceled all in-person registration events through June and leveraged our existing online program for all new students. Canceling inperson events was not something the administration wanted to do since enrollment was already a significant concern and because research suggests that the campus visit is crucial to a student's college decision (Secore, 2018). However, we were in a unique situation to easily transition to a remote format and adjust as necessary to replicate in-person student engagement.

Unlike many schools scrambling to determine a virtual path forward, we already had an online registration program built in our learning management system designed to replicate the in-person offerings. While for families who, for many reasons, could not make an extra visit to campus, the system was uniquely positioned to help us serve all incoming students. By leveraging an existing resource, we could minimize the feelings of missing out and maximize the development of an online cohort experience. Instead of leaving the options open to potentially host some in-person registration days, we demonstrated to students they would all be going through the same online registration program. It was incredibly difficult to cancel all in-person offerings, especially with the overwhelming success they had in fall 2019 and the high registration numbers we already had at that point. Still, creating a shared experience and making a decision that would not need to be changed again mid-stream overshadowed the possibility of holding an in-person program. In hindsight, this was the best decision since very few in-person programs occurred before August 2020. Now, the challenge to hold on to these students without the ability for them to visit campus became our top priority.

\section{We Fear Losing Both Continuing Students and Prospects.}

The registration days and accompanying NSO model were explicitly designed to get students to visit campus and engage with academic advisors earlier, increasing student yield and retention. The decision to 
move the registration day program entirely online, coupled with the uncertainties caused by COVID-10, created a genuine fear of losing our momentum in reversing declining enrollments. Compounding this was the fear that students who were enrolled in spring 2020 just would not come back for the fall term if classes were held remotely or they believed the college experience we could provide was not worth the continued investment. For a school with real enrollment challenges, we were rightly scrambling to figure out how to address this serious concern. As a university, we implemented two critical strategies to retain the incoming cohort of new students and the continuing cohort of current students.

\section{Summer Engagement Series}

Before the COVID-19 pandemic, plans were already in motion to deploy a comprehensive re-recruitment (i.e., retention) initiative for continuing students. These communication strategies would become essential to our ability to increase engagement when students were actively disengaging from their learning and navigating personal adversities presented by the global pandemic.

Initially, the need to highlight landing places or institutional homes for underserved student populations on our campus, such as those with traditionally underrepresented identities, was the impetus for developing a series of summer emails. The idea was to introduce students to places like the Branch Center (our version of a multicultural center), the VETS office, and Disability Services for Students before matriculation, so they both knew and felt comfortable with existing support resources. When the registration day program went entirely online, this need became even more poignant as we were looking at a substantial portion of our students who would be arriving in the fall without ever having set foot on campus. As the impact of these messages shifted, it became our goal to alleviate as much of the anxiety of moving to a new home as possible. We put a face to essentials services in 12 brief emails sent between May and August 2020. These messages were interspersed with six video spotlights filmed and produced by KPCN, a student-run multimedia production team.

Not only was the tone and message of the communications adapting to new needs, but the filming project itself was also forced to adapt to a virtual environment. The KPCN students quickly devised ways for our campus colleagues to film their spotlight pieces remotely and created engaging animations to offset the lower quality of video from recording on a platform like Zoom. Using students who are far more familiar with our target audience allowed us to reach prospective students in ways that both appealed to them and still served as initial introductions to the various campus homes for underserved students on our campus. It also positioned the director of orientation as a new student's primary point of contact throughout the summer months and allowed students to start putting faces to names despite not meeting the staff at an in-person registration day event as planned. The chatter in an unofficial incoming student Instagram account indicated we were promoting the kind of engagement we intended. Followers of the account asked each other, "Did you get the [Orientation Director's Name] email?" - suggesting we were creating critical opportunities for engagement even without the ability to interact with students on campus personally. They began to see we were still here, and we were committed to their successful transition to college. With incoming students hearing from us every week or so, it was also crucial to re-recruit continuing students who may otherwise have considered taking time off to wait out the pandemic or who were at risk of stopping out indefinitely.

\section{Keeping Connected}

Quickly after it was announced in March that all courses would be offered remotely for the remainder of the term and campus offices would be closed for in-person service, a series of resources were made available to the campus community: Keep on Learning, Keep on Working, Keep on Teaching, and Keeping Connected. These online landing pages served as repositories for information and resources, allowing the campus community to continue moving forward despite the seemingly overnight transition to remote learning, working, teaching, 
and engagement. Keeping Connected was developed as an ad hoc group of campus units like the health center, campus dining and housing, campus recreation, and student union and student programming board that were already planning programs for students pretty frequently. The charge for this group was to work together to develop best practices for virtual engagement activities and to help ensure we continued to offer programs through the end of the spring term and eventually through the entire summer. Retention was the primary concern, and it was of the utmost importance to our administration that the campus continued to offer ways for students to connect with their peers so they felt supported and looked after. If we managed to do this, the logic was (Tinto, 1993) our students would enroll for fall classes and return to campus despite the growing uncertainty presented by the COVID-19 pandemic at the time.

Once it became clear that programming would indeed be planned throughout the summer months, the idea to invite and engage incoming students in these virtual events emerged. Our university is a residential campus, serving largely traditional-aged students who primarily take courses in the fall and spring. While we offer summer coursework, most students pursue employment, internships, or simply return to their out-ofstate families during the summer. Until summer 2020, we had never provided much, if any, student-centered programming for continuing students during the downtime, let alone inviting prospective students to join. We leveraged an unofficial incoming student Instagram account and advertised the events directly to many of our new first-year students. We also included all admitted students in the invitation emails going out. In total, we went from zero to eight events over the summer, which were attended in high numbers by new students getting a feel for the university. These events ranged from virtual Office trivia and pet photo competitions to livestreamed haunted campus tours and outdoor movies. By working together as a campus and leveraging existing efforts and resources, we devised a plan that met a variety of student needs when everything was uncertain and addressed the genuine retention concerns of campus administrators.

\section{All Campus Space is Reallocated to Instruction and Limited in Capacity.}

Plans for a fall orientation program seemed to evolve daily in response to constantly changing mitigation guidelines and local and state safety regulations throughout the spring and summer. Following many universities across the country, our statewide system recommended that all campuses adjust their upcoming academic calendars to limit opportunities for students to travel and return to campus. For us, this meant beginning the semester a week and a half early so students would complete finals before the Thanksgiving holiday and would not need to return to campus until spring term in January. The university committed to offering as much inperson instruction as was safely possible, and efforts began to reallocate all available space across campus for instruction, setting up each room with physically distanced tables and chairs, cleaning supplies, and other mitigation protocols. We usually could fit 1,000 people in chairs; we were limited now to 100 at assigned tables, eliminating the possibility of any sort of full-cohort sessions. Virus mitigation protocols drastically and irrevocably altered our ability to host an in-person orientation program designed to accommodate more than 1,000 students at once in a multi-day immersion experience.

With new parameters in place, and the growing economic fallout for local and small businesses, it also became clear fairly early that the community immersion portion of orientation was not going to be feasible, nor would it be reasonable to make such a request to our community partners during a global crisis. The decision was made and communicated to all interested partners that the immersion experience was canceled for fall 2020, with the intention of re-starting the program in fall 2021. All of our collaborators were understanding, agreed it was the right decision, and are on standby to participate again in the future. With one of the most innovative and engaging pieces of our newly redesigned program removed, we had to think quickly about how we would come close to meeting the same learning outcomes for our new class of students. We shifted our focus 
to the things we could control and whittled the program down to the pieces critical to a student's first six weeks of college. It is widely noted (Astin, 1977; Borland, 2016) that if students are successful in those first weeks, they are significantly more likely to persist to the following year and ultimately to graduation. Our task now was to find ways to build connections, integrate students into the culture of their new home, discuss important social expectations and safety measures, and acclimate them to campus.

As the rules for group size and other safety measures continued to evolve through the summer, so did our plans. Ultimately, we ended up running five consecutive days of arrival and move-in leading up to a two-day inperson orientation program. Students were asked to pre-select a move-in date and time and were limited to a few hours during which their two guests could help them load items into the building. During each of these days, the orientation team facilitated tours of campus and the surrounding community, bike rides, drive-through NSO check-in, and evening engagement activities for students. Since only about 200 students were arriving each day, the capacity of these events was not a big concern.

Different measures were put into place for the two-day orientation program, including dividing students into maroon and gray groups and staggering start times. Since no space could accommodate the entire cohort watching our Bystander Intervention Training, for example, this and all other large sessions were pre-recorded. Students were placed into small groups of about 50 based on their academic majors, and within each group, there were 25 maroon students and 25 gray students. Each group was assigned two orientation leaders and a classroom so that at no time were there more than 25 students in a classroom or with a leader. While one color group watched a video, the other color group engaged in an activity or debriefing outside. The leaders would switch the groups back and forth into the classroom for the next video or back outside for their next activity. While this took a significant level of logistical planning and orientation leader training, it seemed the best option for creating mini-cohorts, building community, and providing all the training and information students needed to succeed in their first six weeks of college. The plan worked with only minor hiccups, and overall, students understood the situation and recognized we were doing our best to give them a great experience.

\section{Lessons Learned and Recommendations}

Our institution's experience in adjusting a brand new, highly successful new student orientation program in the face of the COVID-19 pandemic may be unique, but the challenges and constraints presented were not. Colleges and universities worldwide faced similar adversities and were forced to navigate myriad viewpoints, political forces, and state and local safety regulations. The lessons we learned may stem from a unique set of circumstances, but they also speak to shared challenges-many of which continue as we plan to onboard the 2021 cohort of new students. In reflecting on the past year, several lessons and recommendations for the future stand out.

\section{Be Decisive and Stay the Course}

The first important lesson learned in 2020 was to decide a direction based on the current parameters and stick with it as much as possible. When the university closed in March 2020, we had little idea how bad things would get or how long they were going to last. Deciding early to simply cancel all in-person registration day events allowed us to focus on building the best online program we could. Rather than string families along in the hope they would be able to visit campus for an in-person event in May, June, or maybe July, we were able to create a shared online experience. There is camaraderie built in a common struggle. By reframing the program's cancellation as an opportunity for a new online experience, we were able to set the tone and take control of our narrative. While it was not going to be the high-touch retention-focused program it was designed to be, it would still meet our needs in predicting yield and setting students up for success in their first semester of college. It 
also allowed us to lean into the moment and build excitement for the in-person orientation program planned for that fall. Deciding on a course of action focused on students' success despite the restrictions in place ensured success for our programs. It also saved us the consequence of canceling something halfway through and leaving students and families with inequitable onboarding experiences.

\section{Leverage and Build Upon Existing Resources}

In addition to early decision-making, one crucial aspect of our success was leveraging existing resources wherever possible. In the early stages of the pandemic, we began investigating third-party vendors specializing in online orientation and transition programs. It seemed like the right thing to do at the moment-why not trust the experts and invest in a product designed to be remote? We soon realized that we did not need to completely reinvent the wheel because, ideally, we would never be in this situation again. We had an incredibly successful orientation model we would return to when the crisis has passed. We decided to leverage the resources we already had access to and lean on campus colleagues to best use everyone's strengths. It may seem simple, but realizing we already had the tools we needed was a critical turning point for our planning.

Funneling all new students into the existing online registration program we had in Moodle was an easy decision. Subtly shifting the tone of the summer engagement email series was also a natural step. Innovations that emerged included using our enterprise-level Zoom contract to host virtual registration day information sessions that could stream directly to Facebook and asking orientation presenters to use their Zoom accounts to pre-record their sessions rather than trying to figure out how to record them in person safely. We conserved resources by moving all orientation material to a digital format and may never print them again. Another campus department approached us with a speaker they had planned to bring to campus in the spring. We collaborated, using his presentation as a sample academic class rather than creating different class tracks. All institutions face similar challenges. Working together across campus allows us to find mutually beneficial solutions to those challenges.

Breaking down campus silos is perhaps the most significant silver lining to come out of this crisis at our university. The Keeping Connected group developed out of an immediate need to keep students engaged so they would return to campus after remote learning was introduced in spring 2020. This group managed to bring stakeholders from across campus under a common goal-one that continues to unite our campus across silos, academic areas, and cocurricular offerings. Rather than everyone who plans student programs on campus navigating the challenges presented by COVID-19 on their own, we were able to band together and tackle these circumstances as a unified front. Our innovations allowed us to help each other, and the team has become a permanent working group with a newly established mission statement. We are also working to create a campuswide student programming board to continue this work into the future. Silos seem to be a staple of higher education, but leveraging resources and relying on campus colleagues and content experts can eliminate those imaginary barriers and promote program and event development that meets the needs of all students and campus departments.

\section{Create Opportunities for Critical Real-Time Connections}

Another critical lesson learned relates to peer-to-peer, real-time engagement opportunities. Replicating these experiences in the virtual environment or in other ways is essential for helping students make the initial connection to campus. Building on the lessons learned from onboarding the fall 2020 cohort, we are modifying and adjusting our plans for increased student engagement. The pandemic is still a very real threat to the health and well-being of our students, so we are operating under the presumption that the programming limitations we faced in fall 2020 will be similar for fall 2021. With that in mind, our primary focus looking forward is to increase the opportunities students and their families have to interact with university representatives in real- 
time. The online registration day program is already open, with more than 1,000 students signed up to complete it. Students will soon be invited to bi-weekly virtual check-ins where they and family members can simply pop in and ask questions about their next steps. We hope they will feel as though they have access to the university and people who can help. We are working with the campus academic advising community to ensure academic advisors reach out to every new student after online course scheduling. Keeping Connected is hard at work building a full schedule of events for the summer months, again allowing incoming students to meet their future peers much earlier than before. Finding ways to put incoming students face-to-face with their academic home, their incoming classmates, and their upper-class peers is essential at every step in the process. Replicating these moments in a remote environment sets students and the institution up for a promising new cohort of engaged and hopeful students.

\section{Fail Forward and Embrace the Process}

Finally, orientation and transition folks tend to live within a state of constant flexibility and change: Student needs evolve, new institutional leadership requests alterations to existing programs in the 11th hour, and outside forces demand we think quickly on our feet. During a crisis, having a designer's mindset is extremely valuable. Such an approach ensures that carefully thought-out plans and alternatives are available-even if they are never needed. Embracing the design challenge head-on can lead to unsuspected innovations, new campus partners, and retention strategies that may just outlive the current crisis.

\section{Looking Ahead to Fall 2021}

While the world is slowly but surely making progress with vaccination drives and transmission and mortality rates level off, student affairs professionals must begin looking ahead to fall 2021. Even now, we are already starting to onboard our next class of incoming students through online registration programs. Despite the lingering uncertainty, we must make decisions and being executing plans. Our state has made great strides with vaccine distribution, and for all intents and purposes, we are planning to resume regular in-person service to the greatest extent possible. With this in mind, classrooms and campus spaces are still set to accommodate physical distancing, meaning that full-cohort programs may not be possible. The good news is we have been here before. We are confident in planning for a return to normalcy and an orientation program that resembles the one implemented in 2019. If needed, we can quickly scale back to a model that allows for more COVID-19 risk mitigations and smaller group sizes. We plan to re-introduce the community immersion experience as a two-day component of NSO. Conversations with community partners have already begun, and folks seem to be cautiously optimistic about opening up their businesses to new students and families. On all fronts, our message in planning is simple - we hope to return to full-scale new student orientation and are moving forward with plans for that program. We also need to remain flexible and resilient as it may be necessary to scale things down yet again by the time fall rolls around.

\section{Conclusion}

Reflecting on the 2020 orientation cycle has been both cathartic and fulfilling, especially when comparing the devastating feeling we had canceling in-person events and our community immersion program to the outpouring of collaboration and innovation that followed. On the heels of some of the first positive news we had received as an institution in a decade, we faced an impossible challenge presented by a global pandemic. In all honesty, it took a minute for us to set our feelings of defeat aside to realize that we could do this. Just a year earlier, we executed what will still be a marquee program centered around student success and retention. For now, though, there was a new challenge at hand. 
As a medium-sized public flagship institution, we hope what we learned throughout this process can be helpful to other campuses. Whenever possible, OTR professionals make decisions that allow them to employ universal design principles and leave little need to adjust for future safety restrictions. Working across silos, as simple as that sounds, and leveraging existing infrastructure and resources saves precious time and energy, setting the campus community up for long-term success. When in-person programming is not possible, alternative opportunities for real-time peer-to-peer engagement are essential to student retention and persistence. Finally, having solid contingency plans provides peace of mind and allows for a more effective response during a crisis. 


\section{References}

Astin, A. (1977). Four critical years: Effects of College on beliefs, attitudes, and knowledge. Jossey-Bass.

Bauman, D., \& Brown, S. (2019, September 26). The U. of Montana has lost more students this decade than any other flagship. What's going on? Chronicle of Higher Education. https://www.chronicle.com/article/the-uof-montana-has-lost-more-students-this-decade-than-any-other-flagship-whats-going-on/

Borland, K., Jr. (2016). First 100 days persistence-retention plans. Journal of Research, Assessment, and Practice in Higher Education, 1(1), 11-16. https://ecommons.udayton.edu/jraphe/vol1/iss1/6

Secore, S. (2018). The significance of campus visitations to college choice and strategic enrollment management. Strategic Enrollment Management Quarterly, 6(4), 150-158. DOI:10.1002/sem3.20114

St. Amour, M. (2020, October 15). Report: Enrollment continues to trend downward. Inside Higher Ed. https:// www.insidehighered.com/news/2020/10/15/worrying-enrollment-trends-continue-clearinghouse-reportshows

Tinto, V. (1993). Leaving college: Rethinking the causes and cures of student attrition (2nd ed.). University of Chicago Press. 